\title{
Linear embedding via Green's operators: a modeling technique for finite electromagnetic band-gap structures
}

\section{Citation for published version (APA):}

Water, van de, A. M., Hon, de, B. P., Beurden, van, M. C., Tijhuis, A. G., \& de Maagt, P. J. I. (2005). Linear embedding via Green's operators: a modeling technique for finite electromagnetic band-gap structures. Physical Review E - Statistical, Nonlinear, and Soft Matter Physics, 72(5), 056704-1/11. [056704].

https://doi.org/10.1103/PhysRevE.72.056704

DOI:

10.1103/PhysRevE.72.056704

Document status and date:

Published: 01/01/2005

\section{Document Version:}

Publisher's PDF, also known as Version of Record (includes final page, issue and volume numbers)

\section{Please check the document version of this publication:}

- A submitted manuscript is the version of the article upon submission and before peer-review. There can be important differences between the submitted version and the official published version of record. People interested in the research are advised to contact the author for the final version of the publication, or visit the $\mathrm{DOI}$ to the publisher's website.

- The final author version and the galley proof are versions of the publication after peer review.

- The final published version features the final layout of the paper including the volume, issue and page numbers.

Link to publication

\section{General rights}

Copyright and moral rights for the publications made accessible in the public portal are retained by the authors and/or other copyright owners and it is a condition of accessing publications that users recognise and abide by the legal requirements associated with these rights.

- Users may download and print one copy of any publication from the public portal for the purpose of private study or research.

- You may not further distribute the material or use it for any profit-making activity or commercial gain

- You may freely distribute the URL identifying the publication in the public portal.

If the publication is distributed under the terms of Article 25fa of the Dutch Copyright Act, indicated by the "Taverne" license above, please follow below link for the End User Agreement:

www.tue.nl/taverne

Take down policy

If you believe that this document breaches copyright please contact us at:

openaccess@tue.nl

providing details and we will investigate your claim. 


\title{
Linear embedding via Green's operators: A modeling technique for finite electromagnetic band-gap structures
}

\author{
A. M. van de Water, ${ }^{*}$ B. P. de Hon, M. C. van Beurden, and A. G. Tijhuis \\ Department of Electrical Engineering, Eindhoven University of Technology, P.O. Box 513, 5600 MB Eindhoven, The Netherlands \\ P. de Maagt \\ Electromagnetics Division, ESA/ESTEC, P.O. Box 299, 2200 AG Noordwijk, The Netherlands
}

(Received 15 July 2005; published 10 November 2005)

\begin{abstract}
We propose a modular electromagnetic modeling procedure for large finite electromagnetic band-gap (EBG) structures, called linear embedding via Green's operators. It is a diakoptic method based on the HuygensSchelkunoff principle involving equivalent boundary current sources that electromagnetically characterize the enclosed domain of arbitrary shapes, as if it were a multiport system. In a cascade of embedding steps, separate reusable domains are combined to form larger domains. Device design often involves tuning local medium properties in a compact designated domain with a large environment. Through an additional embedding step the equivalent sources describing the environment can be transferred to the boundary of the designated domain, rendering subsequent design steps very fast. This two-stage optimization process is applied in the design of an EBG power splitter.
\end{abstract}

DOI: 10.1103/PhysRevE.72.056704

PACS number(s): 02.70.Pt, 42.25.Fx, 41.20.Jb, 42.70.Qs

\section{INTRODUCTION}

Let us consider an electromagnetic wave that is incident on an aggregate of objects with strong electromagnetic contrasts. If the effective length scale on which the contrast varies, and the wavelength of the field are commensurate, geometric resonance effects may give rise to intricate scattered fields. If the electromagnetic contrast is also periodic, electromagnetic band gaps may occur. Three-dimensional (3D) configurations with one or more band gaps are referred to as electromagnetic band-gap $[1,2](\mathrm{EBG})$ configurations, or, when specifically intended for optical or microwave frequencies, as photonic band-gap and microwave band-gap configurations, respectively. The 3D EBGs have first been proposed in pursuit of the inhibition of spontaneous emission [1], and, in case of carefully prepared disorder in the periodicity, to demonstrate Anderson localization of photons [3].

Following the original interest in these physical phenomena, it was the prospect of producing devices in which electromagnetic fields can be confined in a controlled fashion [4] in one, two and three dimensions via corresponding defects in periodic structures that has sparked extensive EBG research activities over the past decades. For instance, it has been demonstrated, both numerically [5] and experimentally [6] that when an EBG structure is terminated in certain ways, the resulting half-space structure admits surface waves. Local defects inside an EBG structure may give rise to 3D field confinement [7], and right-angle bends joining linear defect waveguides can be tuned such that nearly total transmission is achieved [8]. Many of the EBG structures that have been considered consist of holes or posts in dielectric bulk material or in slabs, providing band-gap field confinement in two dimensions only. The so-called Fan structure [9] and the

\footnotetext{
*Electronic address: A.M.v.d.Water@tue.nl
}

woodpile [10-12] were the first true 3D EBG structures.

The geometric resonance effects not only lead to intricate fields, but also increase the sensitivity of the field to perturbations in the frequency and the scattering geometry. As a consequence, it is possible to design devices, e.g., filters, with a high quality factor, and hence, by definition, a narrow bandwidth. However, the high sensitivity of EBG structures to spatiotemporal changes also implies that it will be hard to achieve a fault-tolerant design, as margins for errors are small. Hence, for analysis and design purposes, it is crucial that in addition to being fast and flexible, the numerical code used for producing the simulations is reliable and accurate.

The so-called plane-wave method has been used extensively for the electromagnetic analysis of fully periodic EBG structures $[13,14]$, and, via the construction of supercells, to emulate the effects of periodic defects [7]. A variety of transfer-matrix methods [15-17] have been developed. These methods are more flexible than the plane-wave method, in that periodicity is no longer required in one direction. However, transfer matrix methods are not inherently stable, in contrast to multiple-scattering-based methods $[18,19]$ for a finite number of scatterers (nonperiodic contrasts) or for periodic contrasts. Dedicated multiple-scattering methods have been developed for spherical scatterers (such as varieties of the vector Korringa-Kohn-Rostocker method [20], for parallel cylindrical scatterers [21-23], and for woodpiles [24].

In the methods mentioned above, the field calculations are performed either directly for the entire configuration, or for single EBG layers that are subsequently stacked, e.g., via the Redheffer star product for scattering matrices [25], to compute the electromagnetic response of a composite-layer structure. For structures with a predominantly planar stratification, the latter approach has two advantages, viz., the intermediate single-layer problems are computationally much smaller than the overall problem, and the resulting single-layer scattering matrices can be reused as building 
blocks for a variety of composite-layer structures. The introduction of an extra field perturbation calculation stage in the scattering-method based Source Model Method provides an efficient way of studying the effects of local cavity defects in a stack of EBG layers [26]. Elements of the method that we propose below are akin to ideas sketched above, in that our method is a multiple-stage method, based on a scattering formalism.

The field scattered in an inhomogeneous region with electromagnetic contrast may be considered as being generated by secondary contrast sources inside, or on the boundary of that region. Invoking Huygens' principle [27], we may consider the scattered field outside a contour (or surface in the $3 \mathrm{D}$, case) surrounding the inhomogeneous region, as having been generated by an equivalent source distribution on that contour (surface). Mathematically, this is referred to as an equivalence principle. The equivalent source distribution is not unique. Love's equivalence principle [28] is based on both electric and magnetic equivalent current sources, whereas Schelkunoff's equivalence principle [29] involves either electric or magnetic equivalent current sources. From the perspective of an observer outside the contour surrounding the inhomogeneous region, that region may be fully characterized electromagnetically through the scattering response via the equivalent source distributions that effectively constitute a multiport system (albeit a continuous one). The decomposition of large systems into interacting multiport subsystems is called diakoptics [30]. Having been used in early days for problems in structural mechanics and electric networks, later diakoptics has been combined with the Method of Moments for the analysis of wire antennas [31,32], and for planar microwave circuits [33]. For these problems the definition of the ports comes naturally. Diakoptics has been applied to field problems in quantum mechanics and electromagnetics through the introduction of a nonlocal admittance operator that provides the boundary conditions for a variational field problem in a confined region of space [34-36]. This method is referred to by the generic term embedding. More recently, diakoptics has been used for supplying boundary conditions for the finite-difference time-domain technique, resulting in the so-called Green's function method $[37,38]$.

We have developed a specific variety of the HuygensSchelkunoff principle based diakoptics for 2D configurations, referred to as linear embedding via Green's operators (LEGO). Embedding has been proposed in inverse scattering $[39,40]$ an alternative to de-embedding, which, like deconvolution, is not stable per se. In its most elementary form, we use embedding to describe multiple scattering between adjacent objects, by considering one of the objects as the environment of the other and vice versa. Instead of likewise decomposing a large field problem into many subproblems that still have to be solved, as one would ordinarily do in diakoptics, we typically start by constructing the linear Green's operators that characterize environments consisting of elementary building blocks, and subsequently employ a cascade of embedding steps to build a library of reusable linear Green's operators that fully characterize various types of complex composite environments. We have reported on some aspects of embedding at conferences [41-43]. Below, we present a full account of embedding, starting with the electromagnetic characterization of an object (or objects) located within a bounded domain by means of a scattering operator. This scattering operator provides a mapping from the equivalent boundary current source distributions, associated with an arbitrary incident field, to equivalent boundary current source distributions associated with the corresponding scattered field. Next, we derive the embedding procedure for a configuration consisting of two disjoint scattering domains, yielding the scattering operator for the combined structure. Subsequently, we discuss the composition of the scattering operator, in case the boundaries of the two original scattering domains have a contour in common. We demonstrate that once the electromagnetic scattering response of a composite structure has been determined, it may be expedient to perform an additional embedding step to transfer the equivalent sources on the outer boundary of the composite structure to equivalent sources on the boundary of a designated domain of interest, which in the case of device design is usually small. Thus, our embedding procedure allows for the separation of the numerical simulations into two parts, viz., incidental time-consuming calculations for the characterization of the large environment, and many fast numerical simulations in the small designated domain, while accounting for the presence of the environment through an environment operator. As an illustration of this two-stage procedure, we consider the optimization of a cavity at the junction of three EBG waveguides in a power splitter.

\section{THE SCATTERING OPERATOR}

As a first step towards embedding, we consider a simply connected bounded 2D domain, containing one or more objects with electromagnetic contrast in a homogeneous background. We want to determine the scattered fields for all possible excitations, on or outside the boundary of the domain. Thus, we capture the complete electromagnetic characterization of the scattering domain in a scattering operator. Inside the scattering domain, any field incident on it may be considered as excited by an equivalent current source distribution on the boundary of the domain. In turn, the scattering operator produces an equivalent current distribution that represents the corresponding scattered field in the exterior domain. The scattering operator relates the incident to the scattered equivalent electric current sources.

To illustrate the scattering operator, we consider the scattering problem depicted in Fig. 1, involving a scattering domain $\mathcal{D}_{1}$ bounded by the contour $\mathcal{C}_{1}$. The scattering object and observation contour may be chosen arbitrarily, as long as the scatterers are enclosed by the contour. For simplicity, we focus on the transversely magnetic polarized cases with $E_{z}$ and $J_{z}$ being the only nonvanishing components of the electric field and (equivalent) electric current, respectively. Henceforth, we drop the subscript $z$. The domain exterior to $\mathcal{D}_{1}$, denoted by $\overline{\mathcal{D}}_{1}$, contains the source distribution that produces an arbitrary incident field $E^{\text {in }}$. Incident plane waves are included implicitly, as being generated by sources at a large distance. Throughout, we reserve the symbol $E^{\text {in }}$ for the incident field in the background medium. 


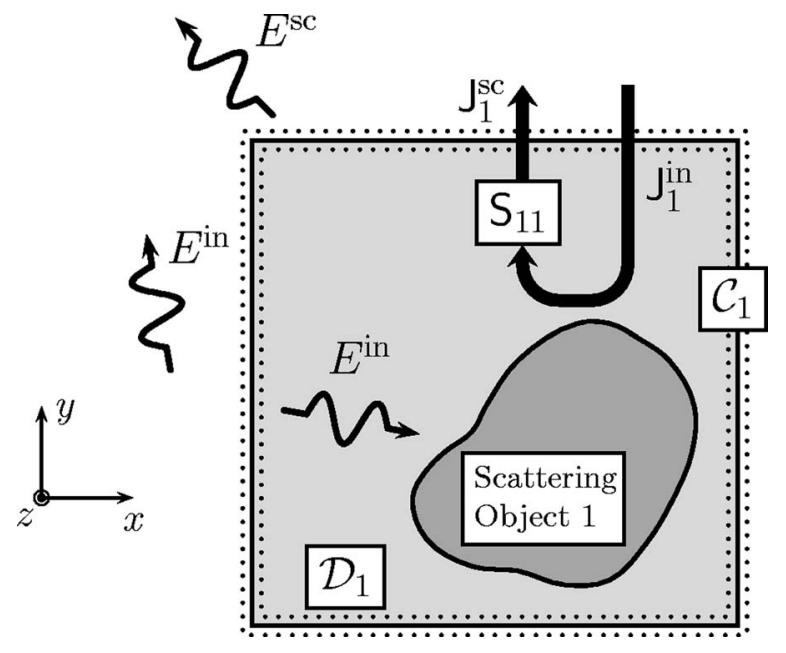

FIG. 1. Scattering by an object in a homogeneous environment using the scattering operator.

To compose the scattering operator of $\mathcal{D}_{1}$, the interior and exterior field problems are separated by application of the Schelkunoff equivalence principle along $\mathcal{C}_{1}$ for both domains $[29,44]$. In this approach, a virtual electrically impenetrable screen is placed at $\mathcal{C}_{1}$, on which equivalent current distributions, $J_{1}^{\text {in }}=J^{\text {in }}(\boldsymbol{\rho})$ and $J_{1}^{\text {sc }}=J^{\text {sc }}(\boldsymbol{\rho})$ for $\boldsymbol{\rho}$ on $\mathcal{C}_{1}$, are defined that, respectively, reproduce the original interior incident and $e x$ terior scattered fields. Mathematically, these current distributions are convolved with the electromagnetic responses of unit-amplitude line sources along $\mathcal{C}_{1}$ for the domain in question. Due to the impenetrable screen, the incident field from $\overline{\mathcal{D}}_{1}$ induces a surface current on $\mathcal{C}_{1}$ that may be regarded as a secondary source, and radiates a field that cancels $E^{\text {in }}$ in $\mathcal{D}_{1}$. The opposite of that current represents the equivalent current distribution, $J_{1}^{\text {in }}$, indicated by the dotted line just inside $\mathcal{C}_{1}$, that would reproduce an identical incident field in $\mathcal{D}_{1}$ (but not in $\left.\overline{\mathcal{D}}_{1}\right)$. Therefore, $J_{1}^{\text {in }}$ is obtained from the electric-field integral equation (EFIE)

$$
-s \mu \int_{\mathcal{C}_{1}} G\left(\boldsymbol{\rho}, \boldsymbol{\rho}^{\prime}\right) J^{\mathrm{in}}\left(\boldsymbol{\rho}^{\prime}\right) d \ell^{\prime}=E^{\mathrm{in}}(\boldsymbol{\rho}), \text { for } \boldsymbol{\rho} \in \mathcal{C}_{1},
$$

where $G$ represents the 2D Green's function defined by

$$
G\left(\boldsymbol{\rho}, \boldsymbol{\rho}^{\prime}\right)=\frac{1}{2 \pi} K_{0}\left(\frac{s\left|\boldsymbol{\rho}-\boldsymbol{\rho}^{\prime}\right|}{c}\right),
$$

and $K_{0}$ denotes the modified Bessel function of the second kind of order zero. The kernel of the integral in Eq. (1) yields the electric field at an observation point $\boldsymbol{\rho}$ due to a unitamplitude electric line source at $\boldsymbol{\rho}^{\prime}$, in a homogeneous medium, with permeability $\mu$ and permittivity $\varepsilon$. Further, $c$ $=(\varepsilon \mu)^{1 / 2}$ and $s$ is taken as $s=j \omega$ where $\operatorname{Re}(\omega) \geqslant 0$ is the radial frequency. To keep the analysis below lucid, we define the propagation operator $P_{1}$

$$
\mathrm{P}_{1}(\boldsymbol{\rho}) J_{1} \hat{=}-s \mu \int_{\mathcal{C}_{1}} G\left(\boldsymbol{\rho}, \boldsymbol{\rho}^{\prime}\right) J\left(\boldsymbol{\rho}^{\prime}\right) d \ell^{\prime}, \text { for } \boldsymbol{\rho} \in \mathbb{R}^{2} .
$$

This operator produces the electric field at $\boldsymbol{\rho}$ due to an electric current distribution $J_{1}$. The subscript of the propagator refers to the contour, $\mathcal{C}_{1}$, on which the current distribution $J_{1}$ is defined. We add an extra subscript to $P_{1}$ for points of observation on $\mathcal{C}_{1}$, i.e., $\mathrm{P}_{11} J_{1}=\mathrm{P}_{1}(\boldsymbol{\rho}) J_{1}$ for $\boldsymbol{\rho} \in \mathcal{C}_{1}$. Accordingly, the EFIE in Eq. (1) is written as

$$
\mathrm{P}_{11} J_{1}^{\text {in }}=E_{1}^{\text {in }} .
$$

Because this equation describes a linear problem, we may formally introduce the inverse propagator of Eq. (3) such that

$$
J_{1}^{\text {in }}=\mathrm{P}_{11}^{-1} E_{1}^{\text {in }},
$$

provided that the corresponding homogeneous equation has only the trivial solution. Although the equivalent current distribution $J_{1}^{\mathrm{sc}}$ that reproduces the exterior scattered field may be obtained directly from the scattered field for a specific incident field via a single EFIE, we prefer using the scattering operator $S_{11}$ instead to encompass the scattered field for all possible excitations. In accordance with Eq. (3), the scattering operator is defined through

$$
J_{1}^{\mathrm{sc}}=\mathrm{S}_{11} J_{1}^{\mathrm{in}}=\int_{\mathcal{C}_{1}} S\left(\boldsymbol{\rho}, \boldsymbol{\rho}^{\prime}\right) J^{\mathrm{in}}\left(\boldsymbol{\rho}^{\prime}\right) d \ell^{\prime} .
$$

The equivalent current distribution $J_{1}^{\mathrm{sc}}$ is depicted by the dotted line just outside $\mathcal{D}_{1}$ in Fig. 1 , since it reproduces the exterior scattered field. To determine the kernel of the scattering operator, $S\left(\boldsymbol{\rho}, \boldsymbol{\rho}^{\prime}\right)$, the scattering behavior of the object inside a homogeneous environment is required for independent source filaments $J_{1}^{\text {in }}$ at sufficiently many sample points $\boldsymbol{\rho} \in \mathcal{C}_{1}$. A source at point $\boldsymbol{\rho}^{\prime}$ generates a field that impinges upon the scattering objects in $\mathcal{D}_{1}$, giving rise to a scattered field $E^{\text {sc }}$. We regard $E^{\text {sc }}$ in $\overline{\mathcal{D}}_{1}$ as if it were generated by secondary contrast sources in a homogeneous background medium. In $\mathcal{D}_{1}, E^{\text {sc }}$ induces a surface current distribution on the virtual electric screen at $\mathcal{C}_{1}$, radiating a field that would cancel $E^{\text {sc }}$ in $\overline{\mathcal{D}}_{1}$. Hence, the opposite of that current is the desired equivalent current distribution $J_{1}^{\mathrm{sc}}$ as defined in Eq. (6). The kernel of the scattering operator then follows from a similar EFIE as in Eq. (5),

$$
S\left(\boldsymbol{\rho}, \boldsymbol{\rho}^{\prime}\right)=\mathrm{P}_{11}^{-1} E_{11}^{\mathrm{sc}},
$$

where the field $E_{11}^{\mathrm{sc}}=E^{\mathrm{sc}}\left(\boldsymbol{\rho}, \boldsymbol{\rho}^{\prime}\right)$ in the kernel of the inverse propagator is the scattered field at $\boldsymbol{\rho}$ on $\mathcal{C}_{1}$ for a source at $\boldsymbol{\rho}^{\prime}$ on $\mathcal{C}_{1}$. The field $E_{11}^{\mathrm{sc}}$ can be determined using conventional methods, e.g., a domain integral equation with second order accuracy as described in [45], or a boundary integral equation (BIE) for dielectric objects [46]. To solve the scattered field numerically for all source positions on $\mathcal{C}_{1}$, a "marching on in source position" scheme [47] may be utilized as a predictor-corrector method to reduce computation times considerably. The total field $E^{\mathrm{t}}(\boldsymbol{\rho})$ is expressed in terms of both equivalent current distributions along $\mathcal{C}_{1}$ according to, 


$$
\begin{cases}\mathrm{P}_{1}(\boldsymbol{\rho}) J_{1}^{\mathrm{in}}+\int_{\mathcal{C}_{1}} E^{\mathrm{sc}}\left(\boldsymbol{\rho}, \boldsymbol{\rho}^{\prime}\right) J^{\mathrm{in}}\left(\boldsymbol{\rho}^{\prime}\right) d \ell^{\prime}, & \boldsymbol{\rho} \in \mathcal{D}_{1}, \\ E^{\mathrm{in}}(\boldsymbol{\rho})+\mathrm{P}_{1}(\boldsymbol{\rho}) J_{1}^{\mathrm{sc}}, & \boldsymbol{\rho} \in \overline{\mathcal{D}}_{1} .\end{cases}
$$

\section{EMBEDDING}

A second scattering object, contained inside a domain $\mathcal{D}_{2}$ and bounded by contour $\mathcal{C}_{2}$, is introduced close to $\mathcal{D}_{1}$ such that the multiple scattering effects between both domains can not be neglected. The embedding procedure accounts for this interaction. It provides the multiple scattering in terms of the scattering operators of the individual domains that have been characterized at an earlier stage. To accomplish this, the domain $\mathcal{D}_{2}$ is considered as part of the environment of $\mathcal{D}_{1}$ and vice versa.

In principle, the equivalent currents that generate the scattered fields exterior to the respective domains are still valid. However, each scatterer produces an additional incident field for the other. We will describe the resulting multiple scattering in terms of a feedback loop involving a modified, "complete" current $J^{\text {cp }}$. By replacing $J^{\text {in }}$ with $J^{\text {cp }}$, the complete field in domain $\mathcal{D}_{1}$ follows immediately from Eq. (8). The combined exterior scattered fields are obtained by application of the scattering operators of both domains via Eq. (6). To convert the incident currents $J^{\mathrm{in}}$, associated with the single scattering domains in absence of the others, into the desired complete current distributions $J^{\text {cp }}$, four $\mathrm{J}$ operators are introduced,

$$
\left[\begin{array}{l}
J_{1}^{\mathrm{cp}} \\
J_{2}^{\mathrm{cp}}
\end{array}\right]=\left[\begin{array}{ll}
J_{11} & J_{12} \\
J_{21} & J_{22}
\end{array}\right]\left[\begin{array}{l}
J_{1}^{\text {in }} \\
J_{2}^{\text {in }}
\end{array}\right] .
$$

Again, from left to right, the subscripts in the operator J, respectively, denote the contours on which the observation and source distributions are located. For example, $J_{12}$ yields an equivalent current distribution on $\mathcal{C}_{1}$ for the complete field in $\mathcal{D}_{1}$ due to the field incident on $\mathcal{D}_{2}$ (in the absence of scattering objects in $\mathcal{D}_{1}$ ). Once the four $\mathrm{J}$ operators have been determined, the entire field problem has effectively been solved.

In order to find expressions for the $\mathrm{J}$ operators, we introduce two current-transfer operators. In Fig. 2, the action of the transfer operator $T_{21}$ is depicted. It produces an equivalent current distribution on $\mathcal{C}_{2}$ for the field in $\mathcal{D}_{2}$ for each possible current distribution on $\mathcal{C}_{1}$. The construction of the transfer operator $T_{21}$ involves two steps. First, the current on $\mathcal{C}_{1}$ generates a field that is propagated to $\mathcal{C}_{2}$, via the propagation operator $P_{21}$. Second, the equivalent current distribution that would produce the corresponding field in $\mathcal{D}_{2}$ is obtained by applying the EFIE via the inverse propagator $\mathrm{P}_{22}^{-1}$ on $\mathcal{C}_{2}$, in a similar fashion as in Eq. (5). This results in

$$
\begin{aligned}
& \mathrm{T}_{21}=\mathrm{P}_{22}^{-1} \mathrm{P}_{21}, \\
& \mathrm{~T}_{12}=\mathrm{P}_{11}^{-1} \mathrm{P}_{12},
\end{aligned}
$$

where a minus sign is concealed in the inverse propagators, as the opposite of the induced currents on each contour re-

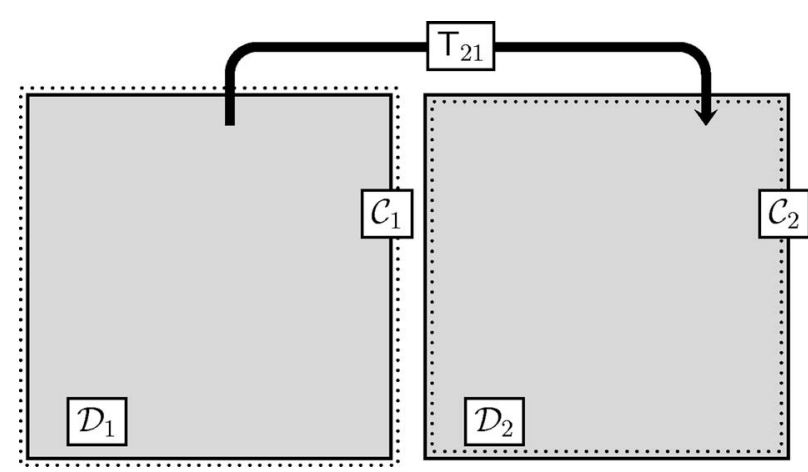

FIG. 2. The current-transfer operator $T_{21}$ transfers current distributions that radiate into $\overline{\mathcal{D}}_{1}$ from the first onto the second contour and subsequently produce the same field in $\mathcal{D}_{2}$.

produce the corresponding interior field in accordance with the equivalence principle. Note that $\mathrm{P}_{22}^{-1}=\mathrm{P}_{11}^{-1}$ for contours with identical shape, and that $P_{21}$ follows from $P_{12}$ by exchanging source and observation contour (reciprocity). Also, when both contours coincide, the transfer operators reduce to identity operators.

In Fig. 3, we have sketched the part of the multiple scattering process that corresponds to an incident field on $\mathcal{C}_{1}$, only. In this case, $\mathcal{D}_{2}$ is considered as the environment of $\mathcal{D}_{1}$. As illustrated in Fig. 3, $J_{11}$ is obtained from $J_{21}$ through scattering in domain $\mathcal{D}_{2}$, with scattering operator $\mathrm{S}_{22}$, and a subsequent transfer of the resulting equivalent current distributions to the first contour via $T_{12}$. However, this does not represent the complete field incident on $\mathcal{D}_{1}$ as the current distribution $J_{1}^{\text {in }}$ also produces a direct contribution in $\mathcal{D}_{1}$, which is accounted for by an additional unit-amplitude identity operator $I_{11}$. The resulting expression for $J_{11}$ thus becomes

$$
J_{11}=I_{11}+T_{12} S_{22} J_{21} \text {. }
$$

Similarly, $J_{21}$ is obtained from $J_{11}$ through scattering via $S_{11}$, and a transfer of the corresponding equivalent currents to $\mathcal{C}_{2}$ via $T_{21}$. Hence, we obtain

$$
\mathrm{J}_{21}=\mathrm{T}_{21} \mathrm{~S}_{11} \mathrm{~J}_{11} \text {. }
$$

For the incident current $J_{2}^{\text {in }}, \mathcal{D}_{1}$ is considered as the environment of $\mathcal{D}_{2}$. Using duality, the $J_{22}$ and $J_{12}$ operators are

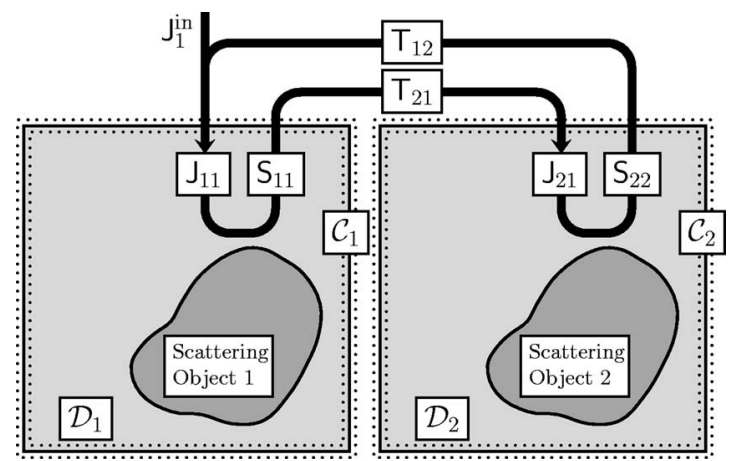

FIG. 3. The $J_{11}$ and $J_{21}$ operators for an incident current $J_{1}^{\text {in }}$ that include the direct incident field and the occurring multiple scattering represented by the loop. 
readily obtained by swapping the indices 1 and 2, thereby exchanging source and observation contour. Combining Eqs. (11) and (12), together with their dual counterparts, yields the following matrix equation:

$$
\left[\begin{array}{ll}
J_{11} & J_{12} \\
J_{21} & J_{22}
\end{array}\right]=\left[\begin{array}{cc}
0 & T_{12} S_{22} \\
T_{21} S_{11} & 0
\end{array}\right]\left[\begin{array}{ll}
J_{11} & J_{12} \\
J_{21} & J_{22}
\end{array}\right]+\left[\begin{array}{cc}
I_{11} & 0 \\
0 & I_{22}
\end{array}\right],
$$

where the last matrix on the right-hand side accounts for the contribution of the incident field in the absence of scatterers in $\mathcal{D}_{1}$ and $\mathcal{D}_{2}$. Next, let us define the reflection operators $R_{11}$ and $\mathrm{R}_{22}$. For $\mathcal{D}_{1}$,

$$
\mathrm{R}_{11}=\mathrm{T}_{12} \mathrm{~S}_{22} \mathrm{~T}_{21} \text {, }
$$

produces equivalent currents on $\mathcal{C}_{1}$ that represent the scattered field from $\mathcal{D}_{2}$ in $\mathcal{D}_{1}$ due to currents on $\mathcal{C}_{1}$. Note that $\mathrm{R}_{11}$ constitutes the Green's operator that characterizes the environment of $\mathcal{D}_{1}$. Likewise, we have

$$
R_{22}=T_{21} S_{11} T_{12} \text {. }
$$

Now, we may express the solution of Eq. (13) in terms of scattering and reflection operators according to

$$
\begin{aligned}
{\left[\begin{array}{ll}
J_{11} & J_{12} \\
J_{21} & J_{22}
\end{array}\right]=} & {\left[\begin{array}{cc}
\left(I_{11}-R_{11} S_{11}\right)^{-1} & 0 \\
0 & \left(I_{22}-R_{22} S_{22}\right)^{-1}
\end{array}\right] } \\
& \times\left[\begin{array}{cc}
I_{11} & T_{12} S_{22} \\
T_{21} S_{11} & I_{22}
\end{array}\right] .
\end{aligned}
$$

The two terms $R_{11} S_{11}$ and $R_{22} S_{22}$ both describe the closed loop as depicted in Fig. 3. The inverse of (I-RS) accounts for the multiple scattering and thus represents the actual embedding of the pertaining domain. The last matrix on the right-hand side contains the incident field contribution of Eq. (13). In particular, $T_{12} S_{22}$ provides the contribution of the incident field on $\mathcal{D}_{1}$ in the absence of scatterers in $\mathcal{D}_{1}$, whereas $T_{21} S_{11}$ provides the contribution of the incident field on $\mathcal{D}_{2}$ in the absence of scatterers in $\mathcal{D}_{2}$. One might think that both embedding operators $\left(\mathrm{I}_{11}-\mathrm{R}_{11} \mathrm{~S}_{11}\right)$ and $\left(\mathrm{I}_{22}\right.$ $-\mathrm{R}_{22} \mathrm{~S}_{22}$ ) have to be evaluated. This is not the case, since the complete incident field in $\mathcal{D}_{1}$, provided by $J_{11}$ and $J_{12}$, is obtained via the embedding operator, $\left(I_{11}-R_{11} S_{11}\right)$, in Eq. (16). Subsequently, the complete incident field in $\mathcal{D}_{2}$, provided by $J_{22}$ and $J_{21}$, follows from $J_{11}$ and $J_{12}$ upon applying Eq. (13). This may be illustrated through the relation,

$$
T_{21} S_{11}\left(I_{11}-R_{11} S_{11}\right)=\left(I_{22}-R_{22} S_{22}\right) T_{21} S_{11},
$$

which follows from

$$
\mathrm{T}_{21} \mathrm{~S}_{11} \mathrm{R}_{11}=\mathrm{R}_{22} \mathrm{~S}_{22} \mathrm{~T}_{21},
$$

and is a direct consequence of the definitions for the reflection operators in Eqs. (14) and (15).

\section{COMPOSITION OF SCATTERING OPERATORS}

With the embedding concept presented above, the interaction between two scattering objects is accounted for. Although the complete interior and exterior total field can be constructed with the obtained $\mathrm{J}$ operators, the procedure still relies on the availability of the incident currents and scattering operators of the individual subdomains. In order to compose a new scattering operator of the combined domain, which may in turn interact with other domains, the embedding procedure will be extended below.

The combined domain $\mathcal{D}_{1} \cup \mathcal{D}_{2}$, is denoted by $\mathcal{D}_{3}$ with outer contour $\mathcal{C}_{3}$. The incident field $E^{\text {in }}$ in $\mathcal{D}_{3}$ is represented by the current distribution $J_{3}^{\text {in }}$, which follows from Eq. (5) for $\mathcal{C}_{3}$. Beware that since we use Schelkunoff's equivalence principle, $J_{3}^{\text {in }}$ cannot be constructed via a mere superposition of the currents $J_{1}^{\text {in }}$ and $J_{2}^{\text {in }}$ used in the previous section. This becomes clear by comparing Eq. (5) for all three contours. The part of $J_{3}^{\text {in }}$ located on $\mathcal{C}_{1}$ thus also contributes to the incident field in $\mathcal{D}_{2}$, even when both domains are not connected. We would like to reproduce this contribution to the incident field in $\mathcal{D}_{2}$ through a corresponding equivalent current distribution on $\mathcal{C}_{2}$. To achieve this, the part of $J_{3}^{\text {in }}$ located on $\mathcal{C}_{1}$ should also be transferred to $\mathcal{C}_{2}$. Hence, the transfer operator $T_{21}$ must be added to the expression for $J_{21}$ in Eq. (13), according to

$$
\left[\begin{array}{ll}
\mathrm{J}_{21} & \mathrm{~J}_{22}
\end{array}\right]=\mathrm{T}_{21} \mathrm{~S}_{11}\left[\begin{array}{ll}
\mathrm{J}_{11} & \mathrm{~J}_{12}
\end{array}\right]+\left[\begin{array}{ll}
\mathrm{T}_{21} & \mathrm{I}_{22}
\end{array}\right],
$$

while $J_{11}$ and $J_{12}$ in Eq. (16) become

$$
\left[\begin{array}{ll}
\mathrm{J}_{11} & \mathrm{~J}_{12}
\end{array}\right]=\left(\mathrm{I}_{11}-\mathrm{R}_{11} \mathrm{~S}_{11}\right)^{-1} \times\left[\mathrm{I}_{11}+\mathrm{R}_{11} \mathrm{~T}_{12}\left(\mathrm{I}_{22}+\mathrm{S}_{22}\right)\right] \text {. }
$$

The dual counterparts of the Eqs. (19) and (20) can be found by swapping indices 1 and 2, but are omitted here for brevity.

When $\mathcal{C}_{1}$ and $\mathcal{C}_{2}$ have no common contour, $\mathcal{C}_{\mathrm{C}}=\mathcal{C}_{1} \cap \mathcal{C}_{2}$ $=\phi$, and hence $\mathcal{C}_{3}=\mathcal{C}_{1} \cup \mathcal{C}_{2}$, the four $\mathrm{J}$ operators immediately represent the $J_{13}$ and $J_{23}$ operators valid for the incident current source distribution $J_{3}^{\text {in }}$. Similarly, the scattering operators of both subdomains form the $S_{31}$ and $S_{32}$ operators. The desired scattering operator $S_{33}$ of $\mathcal{D}_{3}$ then follows directly from the scattering operators of the individual domains,

$$
\mathrm{S}_{33}=\mathrm{S}_{31} \mathrm{~J}_{13}+\mathrm{S}_{32} \mathrm{~J}_{23} \text {. }
$$

In the presence of a common contour $\mathcal{C}_{\mathrm{C}}$, application of the EFIE to $\mathcal{C}_{1} \cup \mathcal{C}_{2}$ indicates that currents induced by an incident field $E^{\text {in }}$ cancel out on $\mathcal{C}_{\mathrm{C}}$. Hence, the contribution from incident currents $J_{\mathrm{C}}^{\text {in }}$ on $\mathcal{C}_{\mathrm{C}}$ may be omitted, which has already been done implicitly in the composition of the $\mathrm{J}_{\mathrm{i} 3}$, with $i$ $=1,2$, operators, as the outer contour $\mathcal{C}_{3}$ excludes common contour parts.

Likewise, we would like to let the scattered field outside $\mathcal{D}_{3}$ be described by equivalent current distributions on the outer contour $\mathcal{C}_{3}$ only. However, there remain equivalent currents on $\mathcal{C}_{\mathrm{C}}$ that contribute to the scattered field in $\overline{\mathcal{D}}_{3}$ and should still be accounted for. To achieve this, the scattering matrices are decomposed into two parts, viz., $\mathrm{S}_{3 \mathrm{i}}$ and $\mathrm{S}_{\mathrm{Ci}}$ with equivalent current distributions on $\mathcal{C}_{3}$ and $\mathcal{C}_{\mathrm{C}}$, respectively. Because the $\mathrm{S}_{\mathrm{Ci}}$ radiate into a homogeneous domain $\mathcal{D}_{3}$, they can be transferred to the outer contour $\mathcal{C}_{3}$ via a transfer operator $T_{3 C}$, in conformity with Eq. (10) (cf. Fig. 4. Hence, 


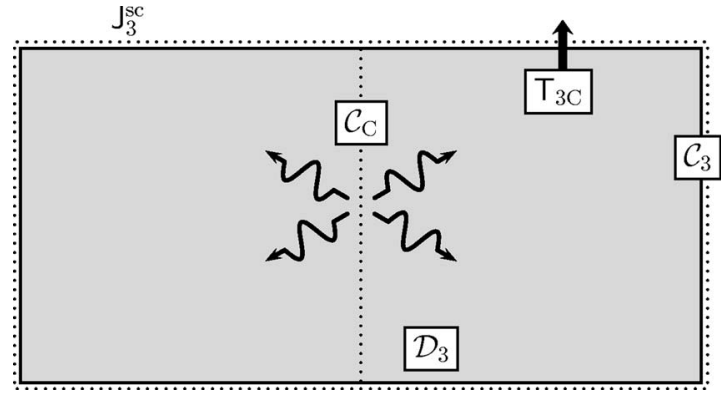

FIG. 4. Both domains with a common contour part, $\mathcal{C}_{\mathrm{C}}$, where equivalent sources that contribute to the scattered field in $\overline{\mathcal{D}}_{3}$ radiate into an empty interior $\mathcal{D}_{3}$.

$$
S_{33}=\left(S_{31}+T_{3 C} S_{C 1}\right) J_{13}+\left(S_{32}+T_{3 C} S_{C 2}\right) J_{23}
$$

represents the scattering operator of the combined domain in the presence of a common contour.

Although both domains are combined into a single scattering object, it remains possible to describe the complete interior fields of a subdomain of interest, say $\mathcal{D}_{i}$, in terms of the incident current $J_{3}^{\text {in }}$ only, i.e.,

$$
J_{\mathrm{i}}^{\mathrm{cp}}=\mathrm{J}_{\mathrm{i} 3} J_{3}^{\mathrm{in}},
$$

in conformity with Eq. (9). The operator $J_{i 3}$ transforms $J_{3}^{\text {in }}$ on the outer contour directly into a $J_{\mathrm{i}}^{\mathrm{cp}}$ on the contour of subdomain $\mathcal{D}_{\mathrm{i}}$. The resulting total field $E^{\mathrm{t}}(\boldsymbol{\rho})$ is given by

$$
\begin{cases}\mathrm{P}_{\mathrm{i}}(\boldsymbol{\rho}) J_{\mathrm{i}}^{\mathrm{cp}}+\int_{\mathcal{C}_{\mathrm{i}}} E^{\mathrm{sc}}\left(\boldsymbol{\rho}, \boldsymbol{\rho}^{\prime}\right) J^{\mathrm{cp}}\left(\boldsymbol{\rho}^{\prime}\right) d \ell^{\prime}, & \boldsymbol{\rho} \in \mathcal{D}_{\mathrm{i}}, \\ E^{\mathrm{in}}(\boldsymbol{\rho})+\mathrm{P}_{3}(\boldsymbol{\rho}) J_{3}^{\mathrm{sc}}, & \boldsymbol{\rho} \in \overline{\mathcal{D}}_{3} .\end{cases}
$$

In a subsequent embedding step $\mathrm{J}_{\mathrm{i} 3}$ can be updated. For instance, the embedding of the previously combined domain $\mathcal{D}_{3}$ with another domain, that changes the outer contour into, say $\mathcal{C}_{4}$, yields a $\mathrm{J}_{34}$ operator, and

$$
\mathrm{J}_{\mathrm{i} 4}=\mathrm{J}_{\mathrm{i} 3} \mathrm{~J}_{34}
$$

replaces $J_{i 3}$ for the new composite structure. The freedom to choose for which of the subdomains $\mathcal{D}_{\mathrm{i}}$ the complete interior field is preserved (and updated) or not, is an additional appealing feature of the embedding procedure.

To assess the corresponding overall storage demand, we consider a large domain $\mathcal{D}_{\mathrm{L}}$ with $N_{\mathrm{L}}$ unknowns (source positions) on its outer contour $\mathcal{C}_{\mathrm{L}}$. $\mathcal{D}_{\mathrm{L}}$ contains $N_{\mathrm{T}}$ previously combined subdomains $\mathcal{D}_{\mathrm{i}}$ with an average number of unknowns $N_{\mathrm{i}}$ on $\mathcal{C}_{\mathrm{i}}$. The associated required amount of storage then becomes $N_{\mathrm{L}}^{2}$ for the $\mathrm{S}_{\mathrm{LL}}$ operator, and $N_{\mathrm{T}} N_{\mathrm{i}} N_{\mathrm{L}}$ for the $J_{i L}$ operators. Although this states the size of both operators explicitly, the size of the combined structure varies substantially with respect to the shape of the outer contour.

As we prefer to relate the memory requirement to the size of the obtained structure, we will provide an upper and lower limit for $N_{\mathrm{L}}$ in terms of the number of previously combined domains $N_{\mathrm{T}}$ and the corresponding average number of unknowns $N_{\mathrm{i}}$ on $\mathcal{D}_{\mathrm{i}}$. In the worst case, when none of the subdomains have contours in common, i.e., $N_{\mathrm{L}}=N_{\mathrm{T}} N_{\mathrm{i}}$, and the size of the operators, $\mathrm{S}_{\mathrm{LL}}$ and $\mathrm{J}_{\mathrm{iL}}$, are proportional to

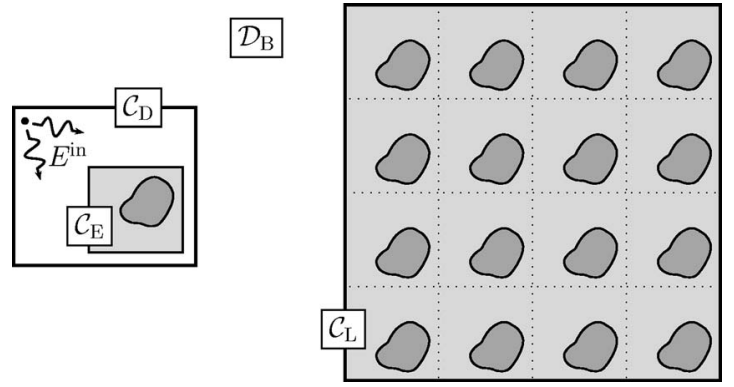

FIG. 5. The designated domain $\mathcal{D}_{\mathrm{D}}$ for subsequent excitation variation and frequent embedding of a domain $\mathcal{D}_{\mathrm{E}}$ with a large scattering domain $\mathcal{D}_{\mathrm{L}}$.

$\left(N_{\mathrm{T}} N_{\mathrm{i}}\right)^{2}$. In the best case, when all subdomains are closely packed together, we have $N_{\mathrm{L}}=N_{\mathrm{i}} \sqrt{N_{\mathrm{T}}}$, and the size of the operators reduces to $N_{\mathrm{T}} N_{\mathrm{i}}^{2}$ for $\mathrm{S}_{\mathrm{LL}}$ and $\mathrm{N}_{\mathrm{T}}^{3 / 2} N_{\mathrm{i}}^{2}$ for $\mathrm{J}_{\mathrm{iL}}$. Of course, the size of $J_{i L}$ reduces further if only the complete interior field of specific subdomains is retained.

\section{OPTIMIZATION THROUGH EMBEDDING}

The theory presented above can be used repeatedly to obtain operators that describe the complete interior and exterior field behavior for large finite structures. By repeated embedding of such a large structure with a sequence of possible inhomogeneous local domains, local structure variations can be studied for optimization purposes.

Because the environment operators encompass all possible excitations, the computation time and the required storage demand will grow considerably for large structures, especially when the interior field of all subdomains is constantly retained. However, both computation time and storage demands can be reduced significantly if the embedding of subsequent domains is restricted to a designated domain, $\mathcal{D}_{\mathrm{D}}$, of interest. Let us elaborate on this via an example illustrated in Fig. 5. Here, a large scattering domain $\mathcal{D}_{\mathrm{L}}$ obtained by repeated application of the embedding procedure is shown. The dotted lines indicate the common contours of the subdomains $\mathcal{D}_{\mathrm{i} ; \mathrm{L}}$ that have been removed at preceding embedding stages. Let us select a designated domain $\mathcal{D}_{\mathrm{D}}$ which is relatively small compared to $\mathcal{D}_{\mathrm{L}}$, and may even be multiply connected. Further, let us stipulate that the outer contour $\mathcal{C}_{\mathrm{D}}$ of $\mathcal{D}_{\mathrm{D}}$ encloses both the source that excites the incident field, and the domains $\mathcal{D}_{\mathrm{E}}$ that are yet to be embedded. This restriction allows us to convert the large scattering operator $\mathrm{S}_{\mathrm{LL}}$ into a reduced scattering operator $\mathrm{S}_{\mathrm{DD}}$ on $\mathcal{C}_{\mathrm{D}}$, where $\mathrm{S}_{\mathrm{DD}}$ reproduces the scattered field of $\mathcal{D}_{\mathrm{L}}$ in $\mathcal{D}_{\mathrm{D}}$ due to current distributions on $\mathcal{C}_{\mathrm{D}}$. Thus we would avoid the timeconsuming direct embedding of $\mathcal{D}_{\mathrm{E}}$ with $\mathcal{D}_{\mathrm{L}}$, which has a large outer contour $\mathcal{C}_{\mathrm{L}}$ by considering the interaction between $\mathcal{C}_{\mathrm{D}}$ and $\mathcal{C}_{\mathrm{L}}$ across $\mathcal{C}_{\mathrm{D}}$ instead, thus significantly reducing computational costs. The composition of $\mathcal{D}_{\mathrm{L}}$ through repeated embedding is the most expensive step, while the subsequent embedding with a designated domain is very cheap, thus facilitating local structure optimization. $\mathrm{T}_{\mathrm{LD}}$, 


$$
\mathrm{S}_{\mathrm{DD}}=\mathrm{T}_{\mathrm{DL}} \mathrm{S}_{\mathrm{LL}} \mathrm{T}_{\mathrm{LD}}
$$

which transfers the interaction from the large outer contour $\mathcal{C}_{\mathrm{L}}$ to the significantly smaller contour $\mathcal{C}_{\mathrm{D}}$. If $N_{\mathrm{D}}$ denotes the number of unknowns on $\mathcal{C}_{\mathrm{D}}$, the complexity of further embedding in $\mathcal{D}_{\mathrm{D}}$ reduces by a factor $\left(N_{\mathrm{D}} / N_{\mathrm{L}}\right)^{2}$. Because $\mathcal{D}_{\mathrm{D}}$ covers an interior embedding approach with respect to $\mathcal{D}_{\mathrm{E}}, \mathrm{S}_{\mathrm{DD}}$ corresponds to the reflection operator for the exterior configuration in Eq. (14). The embedding procedure presented in the previous sections remains valid throughout. After the embedding of $\mathcal{D}_{\mathrm{E}}$ with $\overline{\mathcal{D}}_{\mathrm{D}}$, the complete currents, $J_{\mathrm{E}}^{\text {cp }}$ for $\mathcal{D}_{\mathrm{E}}$ and $J_{\mathrm{D}}^{\mathrm{cp}}$ for $\overline{\mathcal{D}}_{\mathrm{D}}$, include the interaction between $\mathcal{D}_{\mathrm{E}}$ and $\mathcal{D}_{\mathrm{L}}$. They are readily obtained through Eq. (23), from the incident currents $J_{\overline{\mathrm{D}}}^{\text {in }}$ and $J_{\mathrm{E}}^{\text {in }}$ due to a source distribution in $\mathcal{D}_{\mathrm{D}} \cap \overline{\mathcal{D}}_{\mathrm{E}}$ that generates an incident field $E^{\mathrm{in}}$. Introducing $\mathcal{C}_{3}$ $=\mathcal{C}_{\mathrm{D}} \cup \mathcal{C}_{\mathrm{E}}$, we find that the complete field, $E^{\mathrm{t}}(\boldsymbol{\rho})$, in $\mathcal{D}_{\mathrm{D}}$, [cf. Eq. (24)],

$$
\begin{cases}\mathrm{P}_{\mathrm{E}}(\boldsymbol{\rho}) J_{\mathrm{E}}^{\mathrm{cp}}+\int_{\mathcal{C}_{\mathrm{E}}} E^{\mathrm{sc}}\left(\boldsymbol{\rho}, \boldsymbol{\rho}^{\prime}\right) J^{\mathrm{cp}}\left(\boldsymbol{\rho}^{\prime}\right) d \ell^{\prime}, & \boldsymbol{\rho} \in \mathcal{D}_{\mathrm{E}}, \\ E^{\mathrm{in}}(\boldsymbol{\rho})+\mathrm{P}_{3}(\boldsymbol{\rho}) J_{3}^{\mathrm{sc}}, & \boldsymbol{\rho} \in \mathcal{D}_{\mathrm{D}} \backslash \mathcal{D}_{\mathrm{E}} .\end{cases}
$$

Note that only $\mathrm{S}_{\mathrm{DD}}$ is required for the embedding and the reconstruction of the complete field in $\mathcal{D}_{\mathrm{D}}$. Nevertheless, the exterior field in $\mathcal{D}_{\mathrm{B}}$ and the interior field of $\mathcal{D}_{\mathrm{L}}$ can be obtained with relatively low additional costs once the ideal structure is obtained. Because incident currents $J_{\mathrm{D}}^{\text {in }}$ are only present on $\mathcal{C}_{\mathrm{D}}$, the (large) interior field operator $\mathrm{J}_{\mathrm{iL}}$, and scattering operator $\mathrm{S}_{\mathrm{LL}}$ of $\mathcal{D}_{\mathrm{L}}$ can be reduced by a factor $N_{\mathrm{L}} / N_{\mathrm{D}}$, through application of the transfer operator, $\mathrm{T}_{\mathrm{LD}}$, according to

$$
\begin{gathered}
J_{i D}=J_{i L} T_{L D}, \\
S_{L D}=S_{L L} T_{L D},
\end{gathered}
$$

in which $\mathrm{J}_{\mathrm{iD}}$ and $\mathrm{S}_{\mathrm{LD}}$ produce the respective complete interior and exterior fields in $\mathcal{D}_{\mathrm{L}}$ and $\overline{\mathcal{D}}_{\mathrm{D}} \cup \overline{\mathcal{D}}_{\mathrm{L}}$ due to an incident current $J_{\mathrm{D}}^{\text {in }}$. Note that, when $\mathcal{D}_{\mathrm{D}}$ is completely enclosed by $\mathcal{D}_{\mathrm{L}}$, i.e., $\mathcal{C}_{\mathrm{D}}$ is a subcontour of $\mathcal{C}_{\mathrm{L}}$, the transfer operators $\mathrm{T}_{\mathrm{LD}}$ and $T_{D L}$ reduce to identity operators. The complete incident current $J_{\mathrm{i}}^{\mathrm{cp}}$ for each subdomain $\mathcal{D}_{\mathrm{i} ; \mathrm{L}}$ (e.g., the sixteen cells within $\mathcal{C}_{\mathrm{L}}$ in Fig. 5) and the equivalent scattered current $J_{\mathrm{L}}^{\mathrm{sc}}$ are constructed from the complete current $J_{\mathrm{D}}^{\mathrm{cp}}$,

$$
\begin{aligned}
& J_{\mathrm{i}}^{\mathrm{cp}}=J_{\mathrm{iD}} J_{\mathrm{D}}^{\mathrm{cp}}, \\
& J_{\mathrm{L}}^{\mathrm{sc}}=\mathrm{S}_{\mathrm{LD}} J_{\mathrm{D}}^{\mathrm{cp}} .
\end{aligned}
$$

The corresponding total field $E^{\mathrm{t}}(\boldsymbol{\rho})$ in $\overline{\mathcal{D}}_{\mathrm{D}}$ is subsequently obtained from a relation similar to Eq. (27),

$$
\mathrm{P}_{\mathrm{i}}(\boldsymbol{\rho}) J_{\mathrm{i}}^{\mathrm{cp}}+\int_{\mathcal{C}_{\mathrm{i} ; \mathrm{L}}} E^{\mathrm{sc}}\left(\boldsymbol{\rho}, \boldsymbol{\rho}^{\prime}\right) J^{\mathrm{cp}}\left(\boldsymbol{\rho}^{\prime}\right) d \ell^{\prime}, \quad \boldsymbol{\rho} \in \mathcal{D}_{\mathrm{i} ; \mathrm{L}},
$$

$$
\mathrm{P}_{\mathrm{D}}(\boldsymbol{\rho}) J_{\mathrm{D}}^{\mathrm{cp}}+\mathrm{P}_{\mathrm{L}}(\boldsymbol{\rho}) J_{\mathrm{L}}^{\mathrm{sc}}, \quad \boldsymbol{\rho} \in \mathcal{D}_{\mathrm{B}} .
$$

If a subdomain is frequently reused, which is very common for EBG structures $E_{\mathrm{i}}^{\text {sc }}$ and $\mathrm{P}_{\mathrm{i}}(\boldsymbol{\rho})$ can be reused for each subdomain, which accelerates the final field computations considerably.

\section{NUMERICAL VALIDATION}

As a validation of the embedding approach, let us consider two hexagonal unit cells of a 2D triangular EBG with lattice constant $a$, consisting of circular air cylinders inside a dielectric background with relative permittivity $\varepsilon_{r}=11.4$. To operate within the full band gap for both polarizations, of an EBG structure consisting of such cylinders, the frequency is normalized such that $f a / c=0.48$, and the radius of the cylinders is set to $r / a=0.475$. For reference, we employ a BIE with 1250 equivalent electric and magnetic surface currents on each cylinder boundary. The corresponding total electric field strength in Fig. 6(a) where a line source, indicated by a small dot, generates the incident field. To ensure an honest comparison with the theory presented above, we have employed analytical solutions for the fields scattered by a single cylinder. This avoids errors originating from the construction of the initial scattering operator of the individual computational domains. The current distributions on the domain contours used in the embedding approach are discretized with 40 points per wavelength. In Fig. 6(b) we have shown the electric field calculated using the embedding approach. We have highlighted the hexagonal contours $\mathcal{C}_{1}$ and $\mathcal{C}_{2}$. In the scattering operator of the combined structure, the common contour has been removed. Both plots are scaled in $\mathrm{dB}$ to capture possible deviations at different amplitude scales. Nearly no visual difference can be observed between both field plots. Note that the field across the domain contours remains continuous. In this example, a direct advantage of the embedding approach shows up, in that the computational domain contour may be of arbitrary shape, which provides maximum flexibility. For instance, for EBGs with cylinders closely packed on a triangular lattice, the cylinders can not be contained in periodic rectangular computational domains, and hence hexagonal contour shapes would be more suitable.

\section{OPTIMIZATION OF EBG STRUCTURES}

Now, let us apply the embedding approach by considering a power splitter for a single-mode linear-defect waveguide inside a large finite EBG structure. The actual structure is superimposed on the field plots in Figs. 7 and 8. A triangular arrangement of dielectric circular cylinders $\left(\varepsilon_{r}=11.56\right)$ in the air is employed here as a basis. The corresponding periodic EBG structure exhibits a large TM gap about $f a / c=0.407$ for $r / a=0.175$. Within the rectangular supporting structure of 17 by 17 cylinders, three linear defect waveguides are created by removing rows of cylinders. The waveguides are joined at a Y junction through a cavity consisting of a cylinder of arbitrary radius and permittivity surrounded by regular EBG cylinders. In our example, the cavity is tuned to attain high 


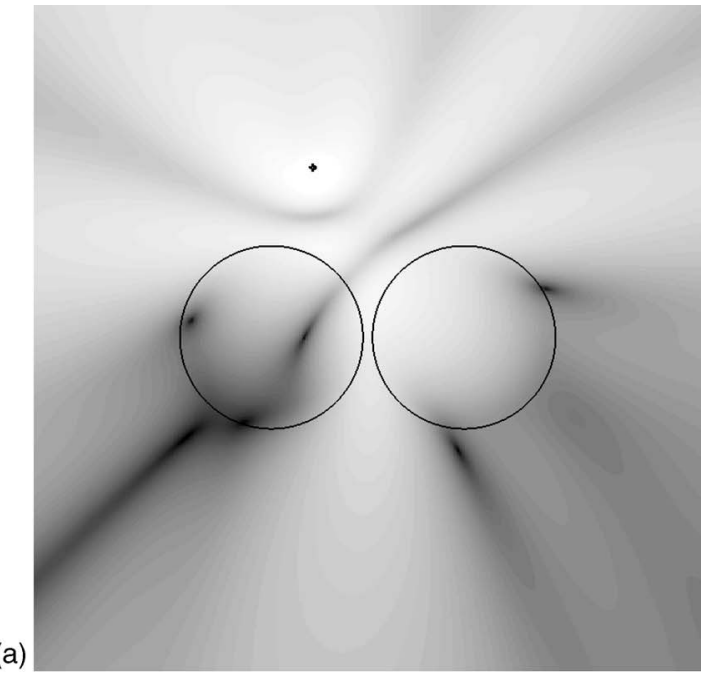

(a)

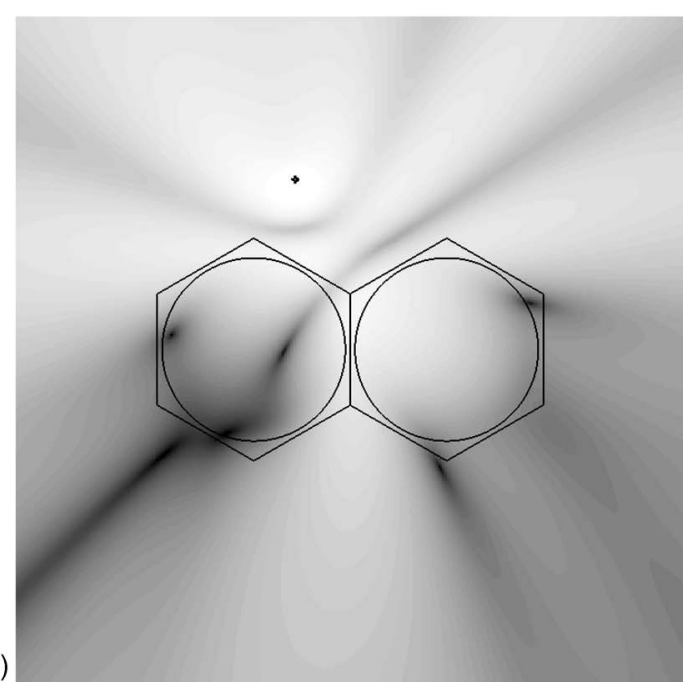

FIG. 6. The total electric field on a logarithmic 60-dB scale of the scattering from two cylinders with the BIE method (a) versus the embedding approach (b). White regions indicate a high field amplitude. The dots indicate the source location.

transmission across the junction, as suggested in [48] for bends.

Due to the relatively small cylinder radius, as compared to the ones in Fig. 6, the shape of the computational domain is not restricted to a hexagonal one. To test the influence of the chosen computational domain, we employ the rhombic shape indicated in Fig. 7 with 18 unknowns on each edge. The EBG structure itself is symmetric with respect to the $x z$ plane (cf. Fig. 1). However, the computational domains are not symmetric, and hence, all asymmetries which occur in the results for a symmetric excitation can be attributed to the embedding approach.

In contrast with popular simulation packages, by which the optimum operating frequency can be determined for a given structure, we are able to optimize the structure for a given operating frequency. In particular, we apply embedding to analyze the effect of different cavity configurations at the $\mathrm{Y}$ junction of the power splitter.

The first stage towards structure optimization is the composition of the scattering operator, $\mathrm{S}_{\mathrm{LL}}$, of the large domain

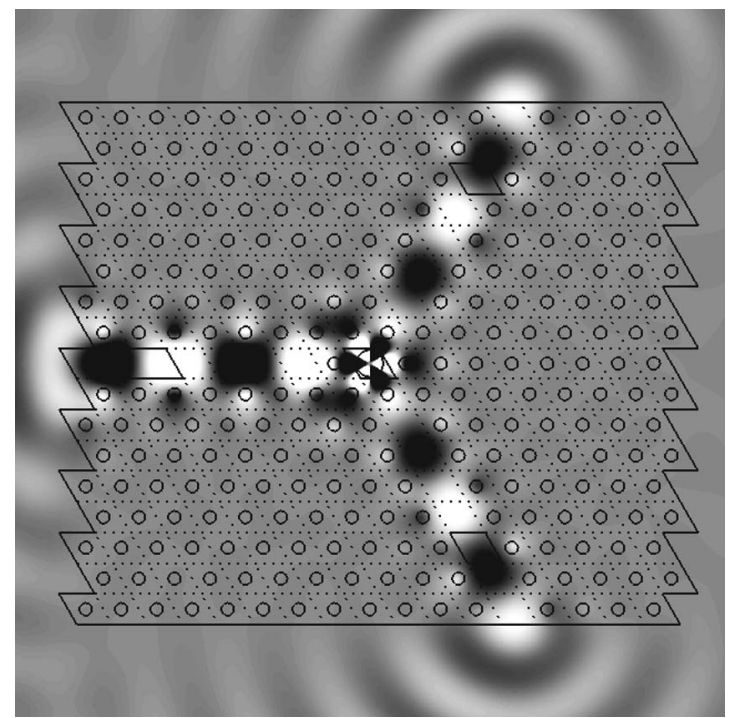

FIG. 7. The total electric field of the hexapole cavity mode with high transmission at $\varepsilon_{r}=28$ and $r / a=0.3767$. The contour $\mathcal{C}_{\mathrm{L}}$ of the large domain $\mathcal{D}_{\mathrm{L}}$ has been highlighted, where the dotted lines indicate removed common contour parts.

$\mathcal{D}_{\mathrm{L}}$ through repeated embedding of reusable scattering domains, starting with a single scattering cylinder. The final outer contour $\mathcal{C}_{\mathrm{L}}$ where $\mathrm{S}_{\mathrm{LL}}$ is defined, is highlighted in Fig. 7. The dotted lines in $\mathcal{D}_{\mathrm{L}}$ indicate common contour parts that have been removed at preceding embedding stages in the composition of $\mathcal{D}_{\mathrm{L}}$. Because $\mathcal{D}_{\mathrm{L}}$ is constructed from single unit cells containing circulair cylinders, the initial scattering operator may still be computed analytically. Although this first stage is very time-consuming, in the order of hours, it is performed only once.

In the initial step of the optimization stage, we assign a relatively small designated domain $\mathcal{D}_{\mathrm{D}}$, which contains the

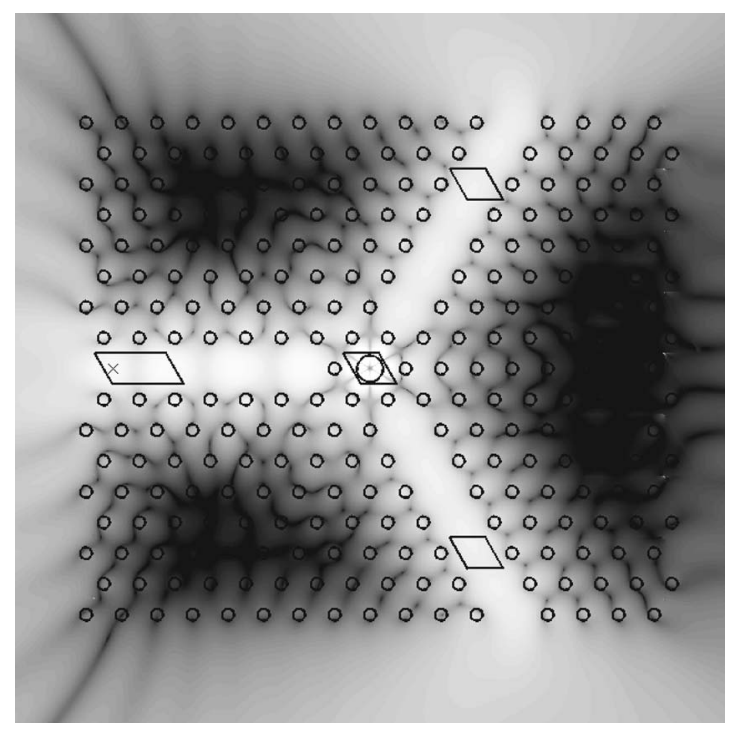

FIG. 8. The total electric field of Fig. 7 on a logarithmic $90-\mathrm{dB}$ scale. White regions indicate a high field amplitude. The contour $\mathcal{C}_{\mathrm{D}}$ of the designated domain $\mathcal{D}_{\mathrm{D}}$ has been highlighted, and the location of the radiating electric line source has been marked by a cross. 


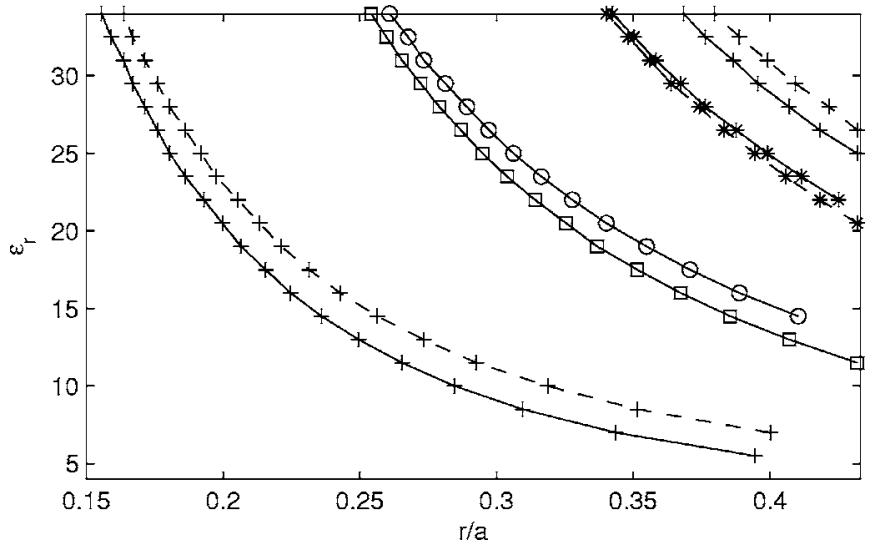

FIG. 9. The resonant cavity modes as a function of $\varepsilon_{r}$ and the radius of the defect cylinder. Solid and dashed lines, respectively, denote local maxima and minima in the transmitted power. $\bigcirc$ : monopole, + : dipole, $\square$ : quadrupole, and $*$ : hexapole.

source and structure variations, as well as the domains where the field values are to be optimized. In the current configuration, this includes the cells containing the defect cylinder inside the cavity, the source in the left waveguide branch, and cells in each of the other two branches where the transmitted power is monitored. The corresponding contour $\mathcal{C}_{\mathrm{D}}$ is highlighted in Fig. 8. On $\mathcal{C}_{\mathrm{D}}$, the reduced scattering operator, $\mathrm{S}_{\mathrm{DD}}$, which describes the scattering response of the large environment in the small domain $\mathcal{D}_{\mathrm{D}}$, is obtained from $\mathrm{S}_{\mathrm{LL}}$ through Eq. (26).

The subsequent optimization stage involves the repeated embedding for a variable $r / a$ and $\varepsilon_{r}$ of the defect cylinder with the large domain $\mathcal{D}_{\mathrm{L}}$. Here, the unit cell that contains the defect cylinder inside the cavity, is taken as the embedding domain $\mathcal{D}_{\mathrm{E}}$. Because each embedding step involves the scattering operator $\mathrm{S}_{\mathrm{DD}}$, instead of $\mathrm{S}_{\mathrm{LL}}$, such an embedding step becomes quite fast, in the order of seconds.

In Fig. 9, the resonant modes of the cavity are shown as a function of $r / a$ and $\varepsilon_{r}$ of the defect cylinder. These modes represent local maxima and minima in the power transmitted through the cavity, respectively, indicated by solid and dashed lines. An electric line source that produces the incident field is positioned in the left waveguide branch in the horizontal symmetry plane of the power splitter. Hence, only even cavity modes are excited with respect to the symmetry plane. The first monopole is not shown, because it occurs for a (nearly) unloaded cavity. The application of scattering operators allows us to vary the source position at each cavity configuration in Fig. 9 as well, without additional costs. Since the optimum remained fairly stable throughout, we have chosen to fix the source position.

A measure of the transmission in terms of the pointing vector in the upper waveguide branch at $\varepsilon_{r}=28$ is presented in Fig. 10. Aside from the first monopole, the local maxima and minima transmission regions appear to be very narrow. The corresponding electric field of the resonant states inside the cavity are shown in Fig. 11. Each maximum, at a dipole or hexapole mode, appears to have a counterpart nearby that produces a minimum. This is caused by the asymmetry of the cavity in the vertical plane (the $y z$-plane in Fig. 1 with re-

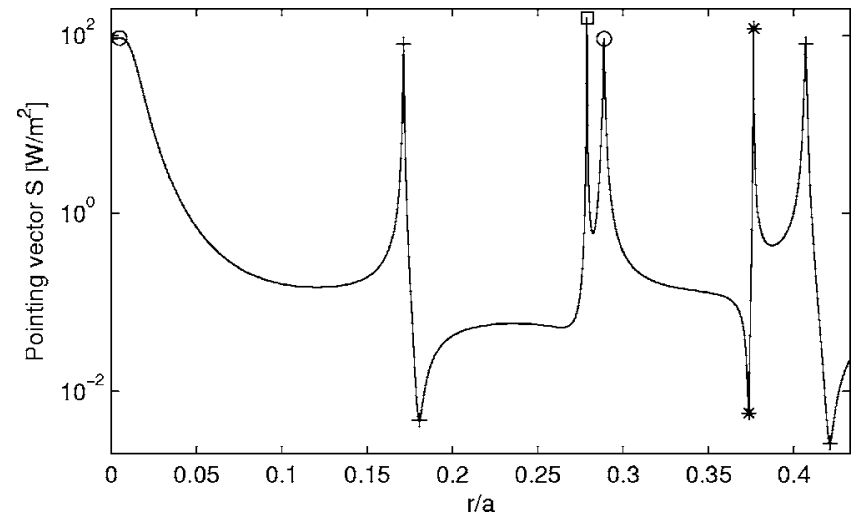

FIG. 10. The pointing vector normal to the cross section of the upper waveguide branch as a function of the defect cylinder radius $r / a$ for $\varepsilon_{r}=28$ excited by an electric line source of $1 \mathrm{~A}$. The markers indicate the cavity modes of Fig. 9.

spect to the center of the cavity) due to the attached waveguide branches. This phenomenon would not be observed if the cavity was placed in an otherwise perfect crystal, which is a common assumption in the analysis of these structures. As a consequence, the vertical asymmetric modes reoccur at nearly the same cavity configuration.

The total electric field in the entire structure for the hexapole mode with a local maximum is depicted in Fig. 7. Despite the vertical asymmetry of the chosen computational domains and building sequence, the obtained field distribution remains symmetric. To capture possible deviations at different amplitudes, the same field distribution is shown in Fig. 8 on a logarithmic $90-\mathrm{dB}$ scale. The symmetry of the field at lower amplitudes deeper within the supporting EBG remains intact. The right-hand side of the structure shows some minor anomalies, but given the amplitude scale and the modest discretization, the results are very accurate.

\section{CONCLUSIONS}

We have proposed an embedding technique based on integral equations and Schelkunoff's equivalence principle that accounts for the multiple scattering between objects in terms of scattering and reflection operators for equivalent current distributions on computational contours. These contours may be of arbitrary shape, which provides maximum flexibility in assembling separate regions into composite structures, for which a new scattering operator can be determined. Results show good agreement with a boundary integral equation method for multiple dielectric objects.

Although embedding is about the exchange of electromagnetic information across the boundaries of separate domains, one may choose to retain the complete interior field information in selected subdomains of interest. Also, the embedding technique is well suited to construct libraries of reusable building blocks, and lends itself for parallel processing as partial substructures can be computed independently.

Once the electromagnetic scattering operator of a large composite structure has been determined through repeated embedding, starting from a single (reusable) building block, 

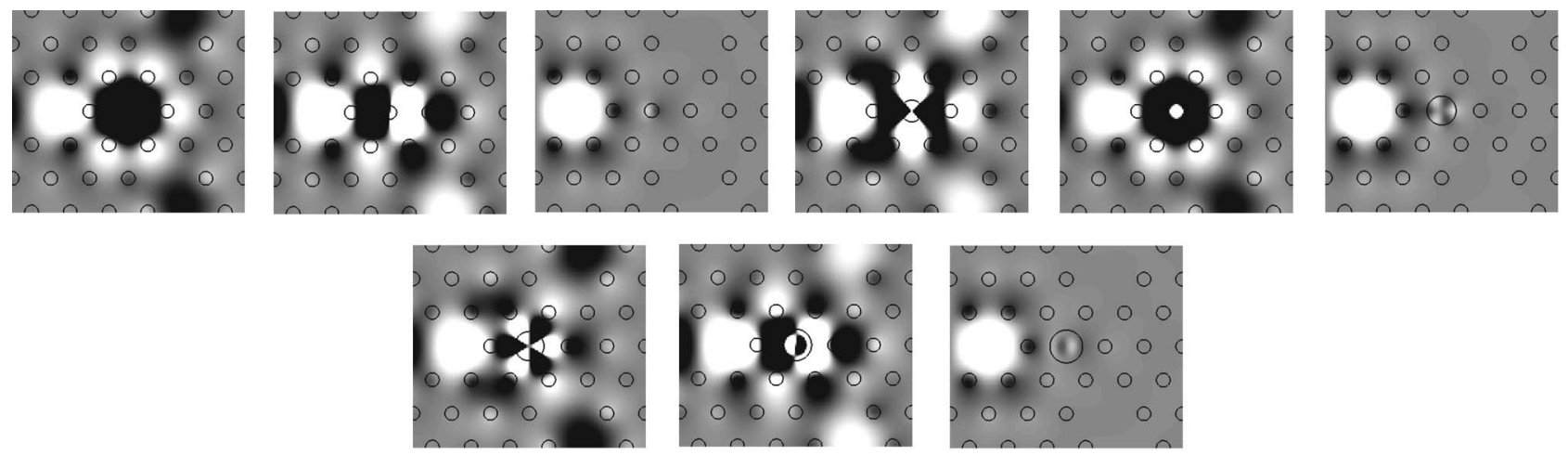

FIG. 11. From left to right and top to bottom the electric field distribution of each of the resonant cavity modes indicated in Fig. 10, at $\varepsilon_{r}=28$ for increasing $r / a$ with the connected waveguide branches included.

it is expedient in performing an additional embedding step to transfer the equivalent boundary sources on the outer boundary of the composite structure to the boundary of a designated domain of interest that encloses the actual sources and the subdomains in which medium variations are considered. In the case of device design this is usually small. Within this designated domain, subsequent embedding with the large composite domain is very cheap, thus facilitating fast local structure optimization. This two-stage process has been demonstrated through the optimization of the transmission characteristics of an EBG power splitter in its dependence on structure variations at the junction of the splitter.
Although the presented theory is applied for finite 2D structures, it may be modified for periodic structures. We are currently implementing LEGO for three-dimensional configurations. Furthermore, fast multipole methods can be applied in the embedding of large structures to reduce computation time.

\section{ACKNOWLEDGMENTS}

The research presented above has been financially supported by ESA/ESTEC, under Contract No. 14897/00/NL/ LvH, and by the Inter-University Research Institute COBRA, under Project No. 99004670.
[1] E. Yablonovitch, Phys. Rev. Lett. 58, 2059 (1987).

[2] A. A. Oliner, in Proceedings of the 29th European Microwave Conference, Munich, Germany, 1999 (Microwave Eng. Eur., London, 1999), Vol. 3, pp. 295-298.

[3] S. John, Phys. Rev. Lett. 58, 2486 (1987).

[4] J. D. Joannopoulos, R. Meade, and J. N. Winn, Photonic Crystals : Molding the Flow of Light (Princeton University Press, Princeton, 1995).

[5] R. D. Meade, K. D. Brommer, A. M. Rappe, and J. D. Joannopoulos, Phys. Rev. B 44, 10961 (1991).

[6] W. M. Robertson, G. Arjavalingam, R. D. Meade, K. D. Brommer, A. M. Rappe, and J. D. Joannopoulos, Opt. Lett. 18, 528 (1993).

[7] R. D. Meade, K. D. Brommer, A. M. Rappe, and J. D. Joannopoulos, Phys. Rev. B 44, 13772 (1991).

[8] A. Mekis, J. C. Chen, I. Kurland, S. Fan, P. R. Villeneuve, and J. D. Joannopoulos, Phys. Rev. Lett. 77, 3787 (1996).

[9] S. Fan, P. R. Villeneuve, R. D. Meade, and J. D. Joannopoulos, Appl. Phys. Lett. 65, 1466 (1994).

[10] E. Ozbay, A. Abeyta, G. Tuttle, M. Tringides, R. Biswas, C. T. Chan, C. M. Soukoulis, and K. M. Ho, Phys. Rev. B 50, 1945 (1994).

[11] M. Bayindir, E. Ozbay, B. Temelkuran, M. M. Sigalas, C. M. Soukoulis, R. Biswas, and K. M. Ho, Phys. Rev. B 63, 081107 (2001).

[12] P. de Maagt, R. Gonzalo, Y. C. Vardaxoglou, and J.-M.
Baracco, IEEE Trans. Antennas Propag. 51, 2667 (2003).

[13] K. M. Ho, C. T. Chan, and C. M. Soukoulis, Phys. Rev. Lett. 65, 3152 (1990).

[14] R. D. Meade, A. M. Rappe, K. D. Brommer, J. D. Joannopoulos, and O. L. Alherhand, Phys. Rev. B 48, 8434(E) (1993); R. D. Meade, A. M. Rappe, K. D. Brommer, J. D. Joannopoulos, and O. L. Alerhand, ibid. 55, 15942 (1997).

[15] P. M. Bell, J. B. Pendry, L. M. Moreno, and A. J. Ward, Comput. Phys. Commun. 85, 306 (1995).

[16] A. Figotin and V. Gorentsveig, Phys. Rev. B 58, 180 (1998).

[17] A. L. Reynolds, H. M. H. Chong, I. G. Thayne, J. M. Arnold, and P. de Maagt, IEEE Trans. Microwave Theory Tech. 49, 2075 (2001).

[18] L. C. Botten, T. P. White, A. A. Asatryan, T. N. Langtry, C. Martijn de Sterke, and R. C. McPhedran, Phys. Rev. E 70, 056606 (2004).

[19] T. P. White, L. C. Botten, Martijn de Sterke, R. C. McPhedran, A. A. Asatryan, and T. N. Langtry, Phys. Rev. E 70, 056607 (2004).

[20] A. Moroz, Phys. Rev. B 51, 2068 (1995).

[21] D. Felbacq, G. Tayeb, and D. Maystre, J. Opt. Soc. Am. A 11, 2526 (1994).

[22] E. Centeno and D. Felbacq, J. Opt. Soc. Am. A 16, 2705 (1999).

[23] A. Ludwig and Y. Leviatan, J. Opt. Soc. Am. A 20, 1553 (2003). 
[24] B. Gralak, M. de Dood, G. Tayeb, S. Enoch, and D. Maystre, Phys. Rev. E 67, 066601 (2003).

[25] R. Redheffer, J. Math. Phys. 41, 1 (1962).

[26] A. Ludwig and Y. Leviatan, J. Opt. Soc. Am. A 21, 1334 (2004).

[27] C. Huygens, Traité de la Lumière (Pierre van der Aa, Leiden, The Netherlands, 1690), composed in 1678.

[28] A. E. H. Love, Philos. Trans. R. Soc. London, Ser. A 197, 1 (1901).

[29] S. Schelkunoff, Bell Syst. Tech. J. 15, 92 (1936).

[30] G. Krohn, J. Appl. Phys. 24, 965 (1953).

[31] C. M. Butler, in IEEE AP-S Int. Symp. Dig. (Dallas, TX, 1990), Vol. 1, pp. 72-75.

[32] A. R. Bretones, R. Mittra, and R. G. Martín, IEEE Microw. Guid. Wave Lett. 8, 281 (1998).

[33] S. Ooms and D. de Zutter, IEEE Trans. Microwave Theory Tech. 46, 280 (1998).

[34] J. E. Inglesfield, J. Phys. C 14, 3795 (1981).

[35] J. E. Inglesfield, J. Phys. A 31, 8495 (1998).

[36] R. Kemp and J. E. Inglesfield, Phys. Rev. B 65, 115103 (2002).

[37] R. Holtzman and R. Kastner, IEEE Trans. Antennas Propag. 49, 1079 (2001).

[38] R. Holtzman, R. Kastner, E. Heyman, and R.-W. Ziolkowski,
IEEE Trans. Antennas Propag. 50, 1017 (2002).

[39] P. M. van de Berg and J. T. Fokkema, IEEE Trans. Microwave Theory Tech. 51, 187 (2003).

[40] A. Franchois and A. G. Tijhuis, Radio Sci. 38, 8011 (2003).

[41] A. G. Tijhuis, M. C. van Beurden, and E. Korkmaz, in 2003 ICEAA International Conference on Electromagnetics in Advanced Applications, Torino, Italy, 8-12 September, 2003, p. 4.

[42] A. M. van de Water, B. P. de Hon, M. C. van Beurden, A. G. Tijhuis, and P. de Maagt, in 2004 URSI International Symposium on Electromagnetic Theory, Pisa, Italy, 23-27 May 2004.

[43] A. M. van de Water, B. P. de Hon, M. C. van Beurden, A. G. Tijhuis, and P. de Maagt, in 2004 27th ESA Antenna Technology Workshop on Innovative Periodic Antennas, Santiago de Compostella, Spain, 8-11 Mar. 2004.

[44] E. J. Rothwell and M. J. Cloud, Electromagnetics (CRC Press, London, 2001).

[45] Z. Q. Peng and A. G. Tijhuis, J. Electromagn. Waves Appl. 7, 739 (1993).

[46] B. de Backer, F. Olyslager, and D. de Zutter, Radio Sci. 32, 1833 (1997).

[47] A. G. Tijhuis, M. C. v. Beurden, and A. P. M. Zwamborn, Elektrik 10, 163 (2002).

[48] Y. Naka and H. Ikuno, in 2004 URSI International Symposium on Electromagnetic Theory, Pisa, Italy, 23-27 May 2004. 